\title{
Histopathological Correlations of Qualitative and Quantitative Temporopolar MRI Analyses in Patients With Hippocampal Sclerosis
}

\author{
Bruna Cunha Zaidan ${ }^{1}$, Ingrid Carolina da Silva Cardoso ${ }^{1}$, Brunno Machado de Campos ${ }^{2}$, \\ Luciana Ramalho Pimentel da Silva'2, Vanessa C. Mendes Coelho², \\ Kairo Alexandre Alves Silveira ${ }^{1}$, Bárbara Juarez Amorim ${ }^{3}$, \\ Marina Koutsodontis Machado Alvim ${ }^{2}$, Helder Tedeschi ${ }^{2}$, Clarissa Lin Yasuda ${ }^{2}$, \\ Enrico Ghizoni ${ }^{2}$, Fernando Cendes ${ }^{2}$ and Fabio Rogerio ${ }^{1 *}$
}

${ }^{1}$ Department of Pathology, School of Medical Sciences, University of Campinas (UNICAMP), Campinas, Brazil, ${ }^{2}$ Department of Neurology, School of Medical Sciences, University of Campinas (UNICAMP), Campinas, Brazil, ${ }^{3}$ Department of

Anesthesiology, Oncology and Radiology, School of Medical Sciences, University of Campinas (UNICAMP), Campinas, Brazil

\section{OPEN ACCESS}

Edited by:

Steven Neal Roper,

University of Florida, United States

Reviewed by:

Daichi Sone,

University College London,

United Kingdom

Emanuele Bartolini,

Fondazione Stella Maris (IRCCS), Italy

*Correspondence:

Fabio Rogerio

fabiorog@unicamp.br

Specialty section:

This article was submitted to

Epilepsy,

a section of the journa

Frontiers in Neurology

Received: 25 October 2021 Accepted: 29 November 2021 Published: 24 December 2021

Citation

Zaidan BC, Cardoso ICS, Campos BM, Silva LRP, Coelho VCM, Silveira KAA, Amorim BJ, Alvim MKM, Tedeschi H, Yasuda CL, Ghizoni E, Cendes F and Rogerio F (2021) Histopathological Correlations of

Qualitative and Quantitative

Temporopolar MRI Analyses in

Patients With Hippocampal Sclerosis.

Front. Neurol. 12:801195

doi: 10.3389/fneur.2021.801195
Hippocampal sclerosis (HS) is a common cause of pharmacoresistant focal epilepsy. Here, we (1) performed a histological approach to the anterior temporal pole of patients with HS to evaluate cortical and white matter (WM) cell populations, alteration of myelin integrity and markers of neuronal activity, and (2) correlated microscopic data with magnetic resonance imaging (MRI) findings. Our aim was to contribute with the understanding of neuroimaging and pathophysiological mechanisms of temporal lobe epilepsy (TLE) associated with HS. We examined MRls and surgical specimens from the anterior temporal pole from TLE-HS patients $(n=9)$ and compared them with 10 autopsy controls. MRls from healthy volunteers ( $n=$ 13) were used as neuroimaging controls. Histological techniques were performed to assess oligodendrocytes, heterotopic neurons, cellular proliferative index, and myeloarchitecture integrity of the WM, as well as markers of acute (c-fos) and chronic $(\Delta$ FosB) activities of neocortical neurons. Microscopic data were compared with neuroimaging findings, including T2-weighted/FLAIR MRI temporopolar blurring and values of fractional anisotropy (FA) from diffusion-weighed imaging (DWI). We found a significant increase in WM oligodendrocyte number, both in hematoxylin and eosin, and in Olig2-stained sections. The frequencies of oligodendrocytes in perivascular spaces and around heterotopic neurons were significantly higher in patients with TLE-HS compared with controls. The percentage of 2',3'-cyclic-nucleotide 3'-phosphodiesterase (CNPase; a marker of myeloarchitecture integrity) immunopositive area in the WM was significantly higher in TLE-HS, as well as the numbers of c-fos- and $\Delta$ FosB-immunostained neocortical neurons. Additionally, we demonstrated a decrease in axonal bundle integrity on neuroimaging, with a significant reduction in the FA in the anterior temporal pole. No differences were detected between individuals with and without temporopolar blurring on visual MRI analysis, considering the number of oligodendroglial cells and percentage of WM CNPase-positive areas. Also, there was no relationship between T2 relaxometry and oligodendrocyte count. 
In conclusion, our histopathological data support the following: (1) the hypothesis that repetitive neocortical neuronal activity could induce changes in the WM cellular constitution and myelin remodeling in the anterior temporal pole from patients with TLE$H S$, (2) that oligodendroglial hyperplasia is not related to temporal blurring or T2 signal intensity on MRI, and (3) that reduced FA is a marker of increase in Olig2-immunopositive cells in superficial temporopolar WM from patients with TLE-HS.

Keywords: epilepsy, hippocampal sclerosis, white matter, immunohistochemistry, MRI, diffusion weighted imaging

\section{INTRODUCTION}

Epilepsy is one of the most common neurological diseases and may be associated with several conditions leading to recurrent epileptic seizures with different presentation patterns (1). In most patients, pharmacological treatment is effective for controlling seizures. However, $20-30 \%$ of individuals do not respond well to antiseizure medications and, therefore, are potential candidates for surgical procedures $(2,3)$. According to the consensus by the International League Against Epilepsy (ILAE), pharmacoresistant epilepsy can be defined as the failure to remit seizures with two adequate regimens of antiseizure medication in either combination or monotherapy (4).

Among the different pathological conditions that cause focal epilepsy, hippocampal sclerosis (HS) is the most frequent histopathological diagnosis in surgical specimens of pharmacoresistant temporal lobe epilepsy (TLE) in adults (5). The pharmacoresistance rate in epilepsy varies depending on the etiology. Specifically, in patients with HS such rate ranges from 75.3 to $90 \%(6,7)$. Morphological changes in the white matter (WM), including oligodendroglial hyperplasia and increased numbers of heterotopic neurons, have been described for decades in TLE surgical specimens (8-10). Additionally, recent experimental studies support the idea that repetitive neuronal activity may induce changes in cell constitution and myelin remodeling in the WM (11), suggesting a maladaptive response.

There are few studies on the correlation of neuroimaging findings with WM microscopic abnormalities in patients with epilepsy. Garbelli et al. (12) performed T2-weighted 7T magnetic resonance imaging (MRI), histological and ultrastructural protocols to investigate surgical samples from individuals with TLE associated with HS and loss of the delimitation between the gray and white matter of the temporal pole on MRI. The authors concluded that such MRI temporopolar blurring on T2-weighted and fluid attenuated inversion recovery (FLAIR) images would be a macroscopic presentation of a long-lasting axonal degeneration and redistribution of the remaining fibers.

In this work, we performed a histopathological, immunohistochemical and neuroimaging approach to the anterior pole of the temporal lobe of patients with HS, with and without MRI T2-weighted/FLAIR temporopolar blurring. These individuals were chosen because HS is a frequent cause of pharmacoresistant focal epilepsy, and no structural change was detected in the neocortical region adjacent to the WM under investigation. Our aim was to study WM changes and associate them with expression of markers of acute and chronic activity in neocortical neurons, thus contributing with the understanding of neuroimaging data (including MRI T2-weighted/FLAIR temporopolar blurring) and with findings supporting pathophysiological mechanisms associated with neuronal activation and WM changes in human TLE-HS specimens.

\section{MATERIALS AND METHODS}

This study was approved by the Research Ethics Committee of our Institution (\#3.087.964) and consisted of retrospective analyses of tissue samples and neuroimaging data from individuals with HS and controls.

Specifically, the cohort of patients with HS $(n=9)$ was the same for both histopathological and MRI evaluations. As an inclusion criteria, we enrolled patients (i) from 2013 to 2018 with clinical diagnosis of pharmacoresistant TLE, MRI signs of HS with and without T2-weighted/FLAIR temporopolar blurring, and a comprehensive preoperative investigation in our tertiary epilepsy center, including EEG, neuroimaging (3 T MRI acquisition, using an 8-channel standard head coil) and neuropsychological examination; (ii) submitted to a temporopolar amygdalohippocampectomy, which led to an en bloc resection of the hippocampus and removal of the anterior temporal pole [according to the formerly published techniques (13)]; (iii) whose surgical histopathological report confirmed the diagnosis of HS [all of them were classified as type I according to the 2013 ILAE proposal (14)] and, (iv) whose paraffin blocks containing the anterior temporal pole specimen were adequately preserved and with tissue amount sufficient to allow for immunohistochemical reactions. Patients who did not fulfill one of the above-mentioned criteria were excluded from the study.

For histopathological evaluation, the control group consisted of adults submitted to autopsy $(n=10)$ with neither neurological disease nor history of seizures. Their anterior temporal pole was sampled as part of a local protocol for central nervous system postmortem evaluation. All autopsy tissues were collected between 6 and $12 \mathrm{~h}$ after death. To avoid interference with the microscopic assessment, only samples in adequately preserved paraffin blocks and that did not show signs of autolysis (eosinophilic cytoplasm and/or pyknotic nuclei) were selected.

For MRI acquisition, the control group consisted of healthy controls $(n=13)$ matched for age and sex, without personal or family history of epilepsy (up to second degree relatives) or other neurologic/psychiatric disorder(s). 


\section{Histological Processing and Immunohistochemical Reactions}

Histological sections ( $4 \mu \mathrm{m}$ thick) were submitted to routine staining (hematoxylin and eosin) or immunohistochemical reactions. For immunostaining, the sections were incubated with primary antibodies for $18 \mathrm{~h}$ at $4{ }^{\circ} \mathrm{C}$ and, then, the detection system containing secondary antibody and peroxidase (AdvanceTMHRP ${ }^{\circledR}$, Dako, cat \# K4068, Glostrup, Denmark) was added for $30 \mathrm{~min}$ at $37^{\circ} \mathrm{C} \cdot 3,3^{\prime}$-Diaminobenzidine (DAB) was used as chromogenic substrate. Specifically, NeuN antibody (1:1000, Merck Millipore $($, cat \# MAB377, A60, Billerica, MA, USA) was employed to evaluate heterotopic neurons. Ki67 marker (1:500, Dako/Agilent ${ }^{\circledR}$, cat \# M7240, MIB-1, Santa Clara, CA, USA) was used to assess cellular proliferative activity. CD34 antibody (1:100, Dako/Agilent ${ }^{\circledR}$, cat \# GA632, QBEnd 10, Santa Clara, CA, USA) was used to detect immature cells. Olig2 staining (1:500, Merck Millipore ${ }^{\circledR}$, cat \# MABN50, 211F1.1, Billerica, MA, USA) was performed to identify oligodendroglial cells. CD45 antibody (1:100, Dako ${ }^{\circledR}$, cat \# GA751, 2B11+PD7/26, Glostrup, Denmark) was used to identify lymphoid cells. Myeloarchitecture integrity was accessed by means of 2', 3'-cyclic-nucleotide 3'-phosphodiesterase (CNPase) immunostaining $\left(1: 500\right.$, cat \# MAB326, 11-5 B, Millipore ${ }^{\circledR}$, Darmstadt, Germany) (15). C-fos (1:100, Abcam ${ }^{\mathbb{R}}$ cat\# ab208942, 2H2, Cambridge-MA, USA) and $\triangle$ FosB (1:100, Abcam ${ }^{\circledR}$, cat \# EPR15905, ab184938, Cambridge-MA, USA) antibodies were used as markers of acute and chronic neuronal activity, respectively (16-19).

For each marker, immunohistochemical reactions were concomitantly performed in all samples to ensure similar technical conditions. External control sections were simultaneously run to confirm the success of the immunostaining.

\section{Histopathological Analysis}

All cell counts in either hematoxylin and eosin-stained or immunostained sections were performed at two levels of the WM, as previously defined (15). Particularly, the superficial white matter (SWM) was defined as $<500 \mu \mathrm{m}$ from the cortical junction, and the deep white matter (DWM) as more than $500 \mu \mathrm{m}$ distant from the cortical junction. For each level (SWM and DWM), 5 randomly chosen highpower fields (HPF; 400x; objective field area equivalent to $0.092 \mathrm{~mm}^{2}$ ) were photographed (Capture system: HD Lite Tucson 1080p; Microscope Novel BM2100). Subsequently, the number of cell types (or immunopositive) was obtained in each photodocumented field using the "cell counter" function of the Image ${ }^{\circledR}$ software (version 1.50i, National Institutes of HealthUS, https://www.nih.gov). The random choice of the histological fields and the cell counts were performed in agreement by two neuropathologists (BCZ and FR).

\section{Analysis of CNPase Immunostaining}

CNPase immunostaining was assessed in slides without counterstaining and employing the Image ${ }^{\circledR}$ software to increase binary contrast (thresholding tool). HPFs of both SWM and DWM levels were randomly selected as described above (item Histopathological Analysis). Briefly, photomicrographs of each HPF were initially transformed into gray scale and then converted into binary information. For such conversion, the software operator visually defined a cutoff point in the gray scale that maintained, as reliably as possible, the immunostaining features observed in the color images (intensity and contrast with the background). Particularly, this gray scale corresponds to numerical values ranging from 0 (black) to 255 (white). Moreover, grayscale values below and above the cut-off point become black and white, respectively. Finally, the software sums the gray values of all pixels in each field and divides by the total number of pixels in the same field, thus, providing a value corresponding to the percentage of immunostained area (\% area) (20).

\section{MRI Acquisition and Processing}

We performed a routine MRI protocol for epilepsy cases in a 3T Philips Achieva scanner, as previously described $(21,22)$. Such protocol included T1-weighted 3D images, T1 and T2weighted images in the axial and coronal planes, fluid-attenuated inversion recovery (FLAIR) images, susceptibility weighted imaging (SWI), inversion recovery (IR) coronal imaging, and diffusion-weighted imaging (DWI). We also performed T2weighted coronal multiecho images for T2 relaxometry analysis (five echo times: 30/60/90/120/150 ms; $3 \mathrm{~mm}$ thick, repetition time $=3300 \mathrm{~ms}$; matrix $200 \times 176$; FOV $1802 \times 180 \mathrm{~mm}^{2}$ ). For diffusion parameter analysis, we acquired a spin-echo single-shot echo planar imaging (EPI) $(2 \times 2 \times 2 \mathrm{~mm}$ voxel size, interpolated to $1 \times 1 \times 2 \mathrm{~mm}$; reconstructed matrix $256 \times 256 ; 70$ slices; TE/TR $61 / 8,500 \mathrm{msec}$; flip angle $90^{\circ}$; 32 gradient directions; no averages; $\max \mathrm{b}$-factor $=1,000 \mathrm{~s} / \mathrm{mm}^{2} ; 6$-min scan) and 3D T1-weighted images $\left(1 \times 1 \times 1 \mathrm{~mm}\right.$, no gap, flip angle $=8^{\circ}$, repetition time $[\mathrm{TR}]=7.0 \mathrm{~ms}$, echo time $[\mathrm{TE}]=3.2 \mathrm{~ms}$, matrix $240 \times 240$, field of view $(\mathrm{FOV})=240 \mathrm{~mm} \times 240 \mathrm{~mm})$.

\section{Relaxometry}

We processed the T2-weighted multiecho coronal images of all individuals with the Aftervoxel software (http://http://www. liv.ic.unicamp.br/ bergo/aftervoxel) as previously documented (21). The region of interest (ROI) was defined using the postoperative T1-weighted image as a visual control to localize the surgical resected area. Then, we manually drew the ROIs in the corresponding topography on the preoperative T2-weighted multiecho, always in the same echo, selecting three portions of the WM in three consecutive slices from approximately the same area of the temporal pole. To define the ROIs in control subjects, we chose an analogous topography to that of the matched patient (anterior temporal pole, ipsilateral to the postoperative area). The ROIs were drawn avoiding areas of artifactual T2 hyperintensity and interference in signal values caused by incorrect demarcation of cerebrospinal fluid in the structures. We carefully drew WM areas of equivalent size across all individuals (Figure 1).

\section{Diffusion Parameters Analysis}

We used DWI and T1-weighted imaging to examine differences in macro and microstructural integrity of the WM. The diffusion maps in the ROIs were obtained using the ExploreDTI 


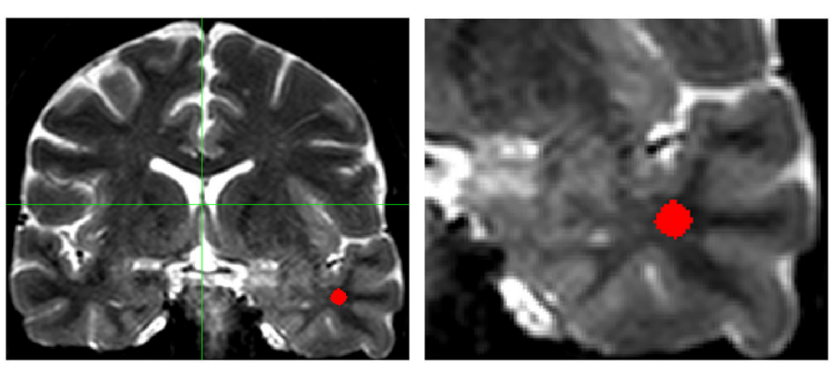

FIGURE 1 | T2-weighted coronal multiecho images showing the localization of the region of interest $(\mathrm{ROI})$ in the white matter of the temporal pole (Left: complete scan, Right: detail of the scan shown on the left). Areas of artifactual increase in T2-weighted hyperintense signal were carefully avoided. Images are in radiological convention.
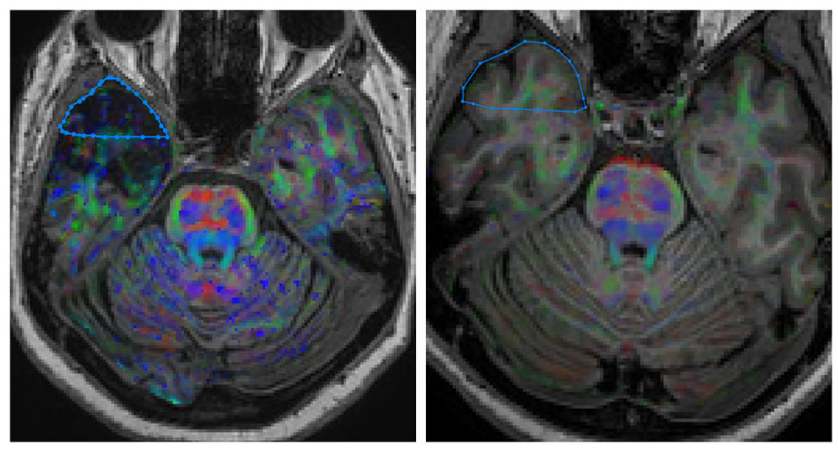

FIGURE 2 | DWI coregistered to the volumetric T1-weighted images showing the localization of the region of interest $(\mathrm{ROI})$ in the white matter of the temporal pole. (Right) HS group; (Left) control group. Images are in neurological convention.

software (A. Leemans, University Medical Center, Utrecht, The Netherlands) (23). First, we registered the preoperative 3D T1-weighted with the postoperative 3D T1-weighted images, using the anterior commissure as an anatomical landmark, as described elsewhere (22). The preoperative DWI was then registered with the resulting coregistered T1-weighted 3D images to ensure the best overlap and define the WM area surgically resected. Then, the axial section where the surgical site presenting the largest dimensions was identified, and the WM ROI was manually drawn. The DWIs were corrected for eddy currents and Gibbs ringing. The susceptibility artifacts were attenuated during non-linear registration using volunteers T1 WI, according to ExploreDTI recommended procedures. We performed the same protocol for controls, defining the ROI in a similar topography, ipsilateral to the surgery of the matched patients. From each ROI, we obtained the mean values of the parameters fractional anisotropy (FA), mean diffusion (MD), axial diffusion (AD), and radial diffusion (RD) (24) (Figure 2).

\section{MRI Analysis}

Magnetic resonance imaging were retrospectively reanalyzed by two experienced neuroimagers (VCMC and FC) considering both clinical and EEG findings. Nonvolumetric sequences were analyzed first with T1 inversion recovery, followed by T1, T2, FLAIR, and SWI sequences. The evaluation corresponded to visual analysis of 12 parameters based on previously described criteria $(25,26)$. We paid particular attention to the presence of blurring and atrophy of the ipsilateral temporal pole, which are reported to occur in $28-66 \%$ of patients with TLE-HS $(12,27$, 28).

\section{Statistical Analysis}

Statistical analyses were performed using SAS 9.2 Software for Windows (SAS Institute Inc, 2002-2008, Cary, NC, USA). The data were analyzed using Fisher exact test for categorical variables and Mann-Whitney test to evaluate differences in MRI variables between groups. Then, we applied receiver operating characteristic (ROC) curves to test whether significant MRI and histopathological metrics were sensitive and specific to alterations in the WM of individuals with TLE-HS. Also, we obtained the best cut-off values optimizing sensitivity and specificity, therefore, indicative of pathological changes. To study the relationship between histopathological and MRI data in the TLE-HS group, Spearman's correlation coefficients were calculated. Finally, we plotted ROC curves to assess whether cell count, immunostaining, and neuroimaging data discriminate TLE-HS group from controls, obtaining a cut-off value associated with a higher probability of changes observed in HS patients. To minimize multiple comparisons, we included in the correlation and ROC analyses the histopathological findings and MRI features considered to be relevant to the group analysis (please refer to the Results section). Data were reported as mean \pm standard deviation (SD), median and interquartile interval, and percentages, as appropriate. The significance level adopted for the statistical tests was $5 \%$.

\section{RESULTS}

\section{Clinical Data}

Nine individuals with ILAE type $1 \mathrm{HS}$ were studied, their ages at surgery ranging from 39 to 56 years old (mean age \pm standard deviation: $47.00 \pm 6.67$ years). Six patients were women $(66.66 \%)$ and three were men $(33.33 \%)$. The average preoperative interval during which seizures occurred was 36.64 years $( \pm 11.02)$, with a minimum of 21 years and a maximum of 50 years. The mean age at seizure onset was 10.4 years $( \pm 11.05)$, ranging from 2 months to 35 years. Seven patients showed fluorodeoxyglucose positron emission tomography (FDG-PET) hypometabolism in the temporal pole (data not available for two patients). Regarding postoperative seizure control [Engel's class (29)], 5 patients (55.55\%) were classified as IA (completely seizure-free since surgery), 1 (11.11\%) was classified as IIA (initially free of disabling seizures but has currently rare seizures), and $3(33.33 \%)$ were grouped as IIIA (worthwhile seizure reduction). The average postoperative follow-up was 2.17 years. Further details on clinical and EEG findings are presented in Table 1.

As regards the control group for histopathological analyses ( $n=10$; five women and five men), the mean age at autopsy 
TABLE 1 | Summary of clinical data and descriptive MRI parameters in patients with HS.

\begin{tabular}{|c|c|c|c|c|c|c|c|c|c|c|}
\hline Patient \# & Gender & $\begin{array}{l}\text { Side of } \\
\text { resection }\end{array}$ & $\begin{array}{l}\text { Age at } \\
\text { onset } \\
\text { (years) }\end{array}$ & $\begin{array}{l}\text { Age at } \\
\text { surgery } \\
\text { (years) }\end{array}$ & $\begin{array}{l}\text { Epilepsy } \\
\text { duration }\end{array}$ & $\begin{array}{l}\text { Engel's } \\
\text { class }\end{array}$ & $\begin{array}{l}\text { Interictal } \\
\text { EEG }\end{array}$ & Ictal EEG & $\begin{array}{l}\text { Temporal lobe } \\
\text { asymmetry }\end{array}$ & $\begin{array}{l}\text { T2/FLAIR } \\
\text { Temporopolar } \\
\text { blurring }\end{array}$ \\
\hline 1 & $\mathrm{~F}$ & Left & 13 & 51 & 38 & la & Left temporal & Left temporal & Present & Absent \\
\hline 2 & $\mathrm{~F}$ & Right & 0.17 & 38 & 37.89 & Illa & $\begin{array}{l}\text { Bilateral } \\
\text { temporal }(\mathrm{R}> \\
\text { L) }\end{array}$ & $\begin{array}{l}\text { Bilateral } \\
\text { fronto-temporal (R } \\
>\mathrm{L})\end{array}$ & Present & Present \\
\hline 3 & M & Left & 2 & 51 & 49 & Ila & Left temporal & $\begin{array}{l}\text { Bilateral temporal } \\
(\mathrm{L}>\mathrm{R})\end{array}$ & Absent & Absent \\
\hline 4 & $\mathrm{~F}$ & Left & 5 & 44 & 39 & la & Left temporal & Left temporal & Present & Present \\
\hline 5 & M & Left & 3 & 49 & 46 & Illa & $\begin{array}{l}\text { Bilateral } \\
\text { temporal }(L> \\
\text { R) }\end{array}$ & $\begin{array}{l}\text { Bilateral temporal } \\
(L>R)\end{array}$ & Present & Present \\
\hline 6 & $\mathrm{~F}$ & Right & 18 & 41 & 23 & la & $\begin{array}{l}\text { Right } \\
\text { temporal }\end{array}$ & Right temporal & Absent & Absent \\
\hline 7 & $\mathrm{~F}$ & Right & 35 & 56 & 21 & Illa & $\begin{array}{l}\text { Bilateral } \\
\text { temporal }(\mathrm{R}> \\
\text { L) }\end{array}$ & Right temporal & Absent & Absent \\
\hline 8 & $\mathrm{~F}$ & Left & 4 & 54 & 50 & la & $\begin{array}{l}\text { Bilateral } \\
\text { temporal }(L> \\
\text { R) }\end{array}$ & Left temporal & Absent & Present \\
\hline 9 & $\mathrm{M}$ & Left & 13 & 39 & 26 & la & Left temporal & Left temporal & Absent & Present \\
\hline
\end{tabular}

was $43.00 \pm 12.38$ years (ranging from 23 to 67 years). For MRI control group ( $n=13$; eight women and five men), the mean age at acquisition was $52.54 \pm 8.96$ years (ranging from 36 to 65 years). There was no statistical difference regarding age when the HS group was compared to autopsy and MRI controls $(p=0.326$ and $p=0.101$, respectively).

\section{Quantitative Analysis of Cell Population in the White Matter}

Hematoxylin and eosin-stained slides from the anterior pole of the temporal lobe from individuals with HS showed a significant increase in the number of oligodendroglial cells per HPF, both in the SWM and DWM, in comparison with controls (HS SWM: 173.53 (mean) \pm 27.87 (standard deviation) and DWM: $159.20 \pm 21.56$; Control (C) - SWM: $97.88 \pm 14.01$ and DWM: $95.56 \pm 16.06 ; p<0.001$ ) (Figure 3). Such increase was also detected using the Olig2 antibody (HS - SWM: $135.02 \pm 19.97$, DWM: $105.31 \pm 16.52$; C - SWM: $46.82 \pm 15.78$, DWM: 36.80 $\pm 10.42 ; p<0.001$ ) (Figure 4). In addition, the HS group presented a significant increase in the number of perivascular oligodendroglial cells per vessel (HS - 28.09 \pm 17.14 ; C $-0.16 \pm$ $0.39 ; p<0.001$ ) or adjacent to heterotopic neurons (perineuronal satellitosis; HS - SWM: $1.96 \pm 2.75$, DWM: $0.64 \pm 0.55$; C SWM: $0.08 \pm 0.19 ; p=0.008$, DWM: $0.04 \pm 0.13 ; p=0.001)$ (Figure 5).

As regards heterotopic neurons, we observed an increase in their frequency in the SWM of HS samples only after labeling with NeuN in comparison with controls (HS - SWM: $1.47 \pm 0.46 ; \mathrm{C}-\mathrm{SWM}$ : $0.70 \pm 0.29 ; p=0.002$ ) (Figure 6). Particularly, no significant difference was noted in such cell count on hematoxylin and eosin-stained sections both in SWM (HS $-0.71 \pm 0.66 ; \mathrm{C}-0.28 \pm 0.21 ; p=0.171$ ) and DWM (HS -
$0.33 \pm 0.36 ; \mathrm{C}-0.10 \pm 0.22 ; p=0.051)$. Likewise, there was no difference between the groups regarding the number of NeuNpositive neurons in the DWM ( HS - $0.47 \pm 0.22$; C $-0.32 \pm 0.30$; $p=0.151)$.

Neither chronic inflammation nor CD45-positive cells were identified in the groups. Immunostaining for CD34 was verified only in the vascular endothelium (no immature CD34-positive cells were observed), irrespective of epilepsy history.

\section{Tissue Expression of CNPase and Ki67 Labeling}

The percentage of CNPase-immunopositive area was significantly higher both in the SWM and DWM in patients with HS compared with controls (HS - SWM: $28.51 \pm 9.58 \%$, DWM: $25.80 \pm 10.06 \%$; C - SWM: $7.66 \pm 4.35 \%$, DWM: $7.02 \pm 3.09 \%$; $p<0.001$ ) (Figure 7).

Proliferative activity, as shown by nuclear staining for Ki67, was significantly higher in the DWM of the HS group (HS - 0.44 $\pm 0.42, \mathrm{C}-0.04 \pm 0.13 ; p=0.005)$, but not in SWM (HS -0.18 $\pm 0.27, \mathrm{C}-0.02 \pm 0.06 ; p=0.091)$.

\section{Tissue Expression Analysis of Acute and Chronic Neuronal Markers}

We also studied markers of acute (c-fos) and chronic ( $\Delta$ FosB) neuronal activity in both the groups. Specifically, there was a significant increase in neocortical immunolabeling for c-fos (HS $-0.57 \pm 0.77 ; \mathrm{C}-0.12 \pm 0.18 ; p=0.004)$ and $\Delta$ FosB (HS -13.86 $\pm 7.54 ; \mathrm{C}-0.07 \pm 0.13 ; p<0.001$ ) in patients with HS (Figure 8).

\section{MRI Findings}

The parameters assessed in MRI scans and their corresponding frequencies in the HS group are depicted in Table 2. Specifically, 

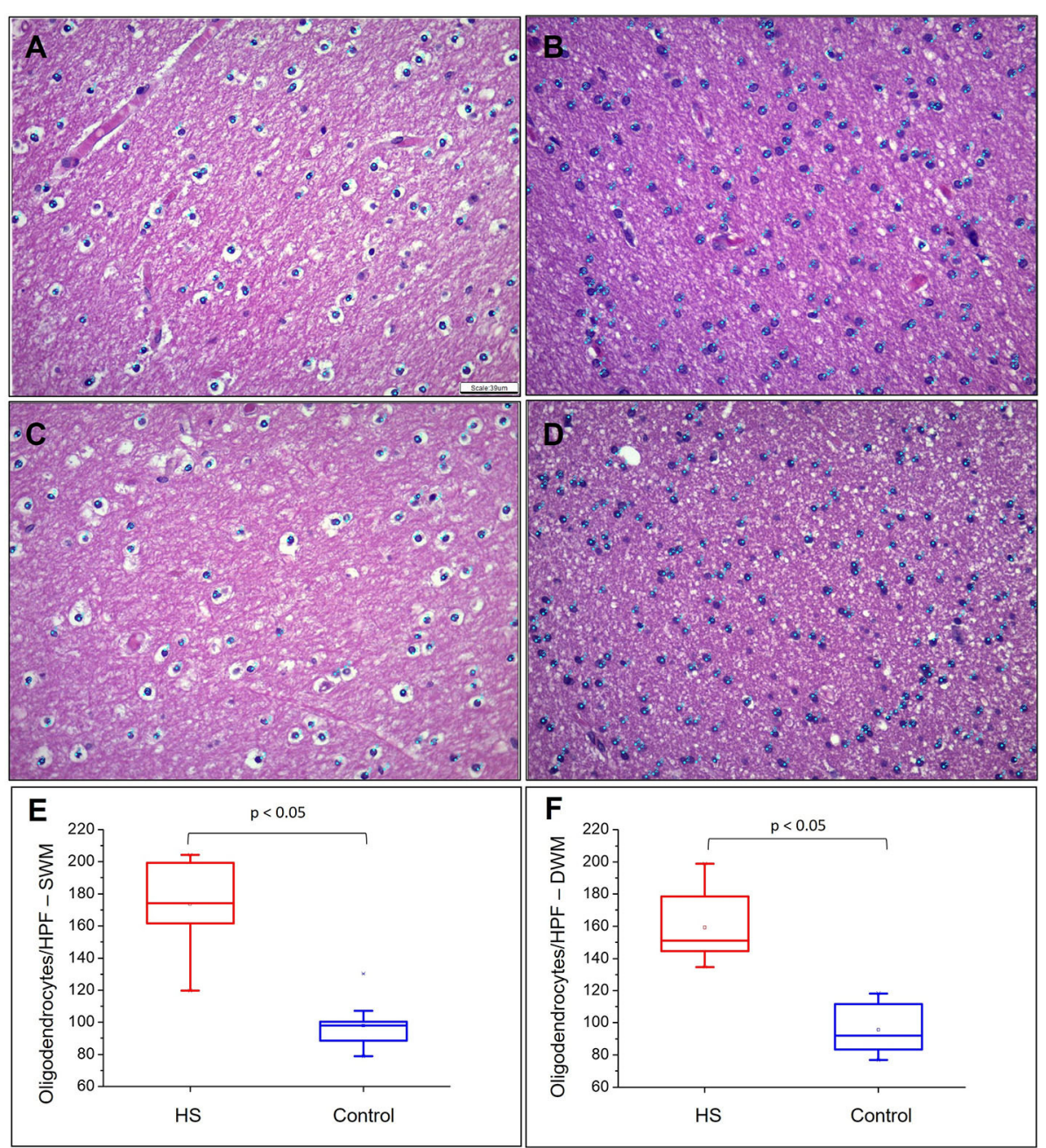

FIGURE 3 | Quantitative analysis of the number of oligodendrocytes in the superficial white matter (SWM) (A) control and (B) HS group; and in the deep white matter (DWM) (C) control and (D) epilepsy group. Graphical representation of the mean number of oligodendroglial cells in SWM (E) and DWM (F). Note the identification of cells counted in each field by means of blue dots corresponding to the "cell counter" counting tool of the ImageJ® software. Hematoxylin and eosin (A-D). Scale bar: $39 \mu \mathrm{m}(\mathbf{A}-\mathbf{D})$

there was a significant increase in T2 signal values (relaxometry) in patients with epilepsy when compared with controls $(p=$ $0.002)$. Regarding DWI, we found significantly reduced FA values in the HS group ( $p=0.034)$, but no significant differences for AD $(p=0.185), \mathrm{RD}(p=0.064)$, or $\mathrm{MD}(p=0.093)$.

All patients had classical MRI signs of unilateral HS in the visual analysis (Figure 9). Temporopolar blurring in T2weighted/FLAIR images was present in five out of nine patients. Temporal lobe asymmetry was observed in four out of nine patients. One patient also had a small encephalocele.

\section{Analyses of Association Between Clinical Data, Cell Count, MRI Findings, and Immunohistochemical Expression}

A positive correlation was found between the number of cells expressing the chronic neuronal activity marker $(\Delta \mathrm{FosB})$ in the cortex and the number of Olig2-positive cells in the DWM ( $r=$ $0.700 ; p=0.036$ ) (Figure 10).

Moreover, there was a negative correlation between the number of Olig2-positive cells and the FA in the SWM $(r=$ $-0.666 ; p=0.049)$ and a non-significant correlation in DWM $(r=-0.250, p=0.51)$ (Figure 10). We also found a negative correlation between the number of cells expressing $\triangle F$ FosB in the SWM and the FA mean in patients with HS $(r=-0.788 ; p$ $=0.012$ ).

There were no significant correlations between oligodendrocyte count (hematoxylin and eosin-stained sections) and T2-weighted signal measured by relaxometry in both the SWM $(-0.083, p=0.83)$ and DWM $(r=-0.500, p=0.17)$; oligodendrocyte count and FA in the SWM $(r=-0.0833, p$ $=0.83)$ and DWM $(r=-0.183, p=0.63)$; CNPase-positive area (\%) and T2 signal in the SWM $(r=0.017, p=0.96)$ and DWM $(r=-0.283, p=0.46)$; and CNPase-positive area and 


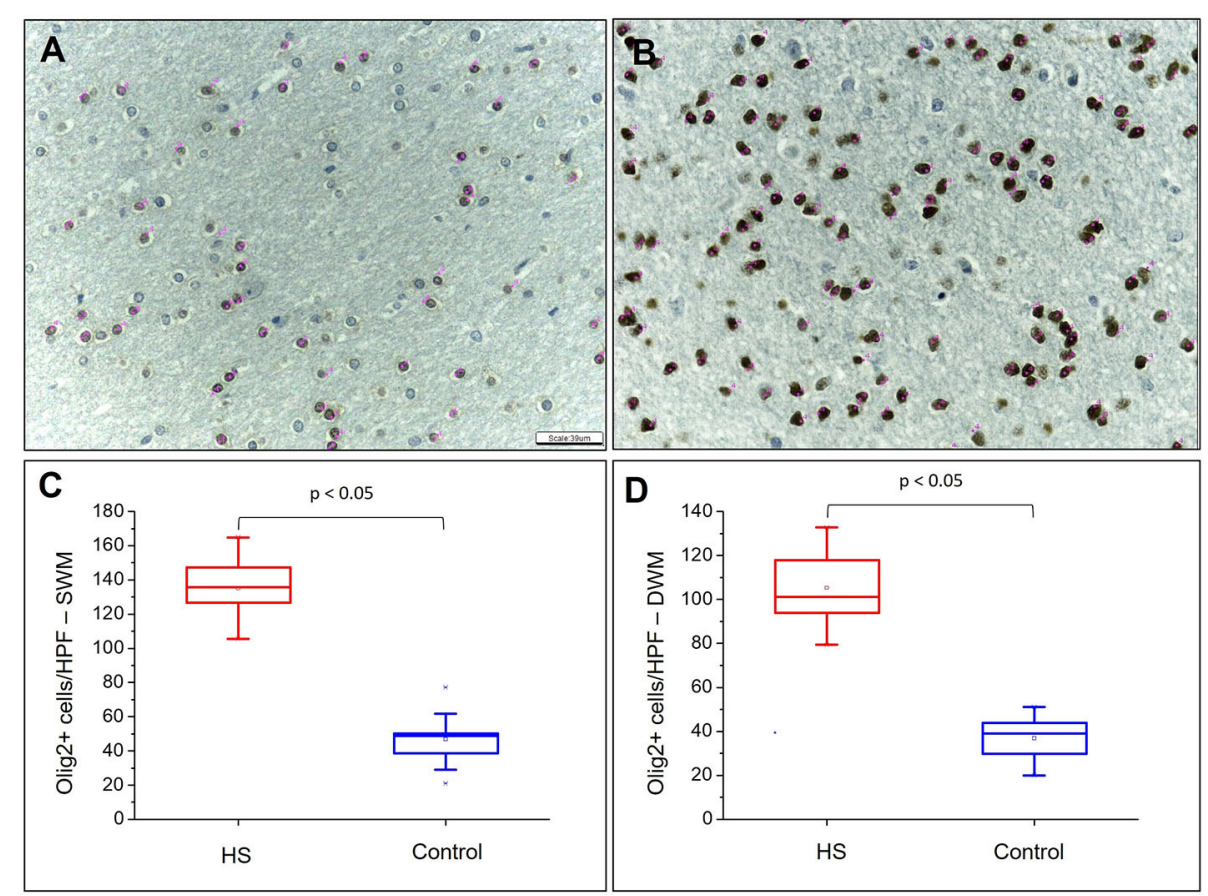

FIGURE 4 | Quantitative analysis of the immunohistochemical expression of Olig2. Olig2 expression in control (A) and HS (B) individuals. Graphic representation of the average number of cells with Olig2 expression in SWM (C) and DWM (D). Note the identification of cells counted in each field by means of pink dots corresponding to the "cell counter" counting tool of the ImageJ® software. Peroxidase (A, B). Scale bar: $39 \mu \mathrm{m}$ (A, B).

FA in the SWM $(r=0.317, p=0.41)$ and DWM $(r=0.001$, $p=1.00)$.

When comparing patients with and without temporal polar blurring in the visual analysis of T2-weigthed and FLAIR images, we did not find significant differences $(p>0.1)$ in the number of oligodendroglial cells (hematoxylin and eosin-stained sections and Olig2-positive) and CNPase-immunopositive areas. Moreover, there were no significant correlations ( $p$ $>0.1$ ) when considering the number of oligodendroglial cells or CNPase-immunopositive areas and duration of epilepsy.

\section{Discrimination of Study Groups Using ROC Curves}

The ROC analyses showed that the number of oligodendroglial cells (hematoxylin and eosin-stained sections) differentiated patients with HS both in the SWM (AUC $=0.989$, 95\%CI $0.954-1.024 ; p<0.001)$ and DWM (AUC $=1$, 95\%CI $1-$ $1 ; p<0.001)$. Particularly, the cut-off point for cells in the SWM was $\geq 113$ cells/HPF with sensibility $=100.0 \%$ (95\%CI 62.88-100.0) and specificity $=90 \%$ (95\%CI 54.1199.48). Regarding the DWM, the cut-off point was $\geq 126$ cells/HPF with sensibility $=100.0 \%(95 \% \mathrm{CI} 62.88-100.0)$ and specificity $=100 \%(95 \%$ CI $62.55-100.0)$. Similarly, the number of oligodendroglial cells in the perivascular space $(\mathrm{AUC}=1$, $95 \%$ CI $1-1 ; p<0.001$ ) differentiated the HS group. In this case, the cut-off point was $\geq 6$ cells/vessel with sensibility $=100.0 \%$
$(95 \%$ CI $62.88-100.0)$ and specificity $=100.0 \%(95 \%$ CI $65.55-$ 100.0).

As regards immunostaining, we found that the number of NeuN-positive heterotopic neurons in the SWM differentiated the HS group (AUC $=0.917$, 95\%CI $0.783-$ $1.050 ; p<0.002$ ) with a cut-off point $\geq 1.3$ NeuN-positive cells/HPF with sensibility $=77.78 \%$ (95\%CI $40.19-96.05)$ and specificity $=100.0 \%(95 \%$ CI $65.55-100.0)$. Also, Olig2positive cells differentiated patients with $\mathrm{HS}$ both in the SWM $(\mathrm{AUC}=1,95 \% \mathrm{CI} 1-1 ; p<0.001)$ and in the DWM $(\mathrm{AUC}=1$, 95\%CI 1-1; $p<0.001$ ), cut-off points being $\geq 91$ cells/HPF with sensibility $=100.0 \% \quad(95 \% \mathrm{CI}$ $62.88-100.0)$ and specificity $=100.0 \% \quad(95 \% \mathrm{CI} \quad 65.55-$ $100.0)$; and $\geq 65$ cells $/$ ) and specificity $=100.0 \%(95 \% \mathrm{CI}$ 79.95-100.0), respectively.

The percentage of area immunoreacted to CNPase also differentiated the HS group, both in the SWM (AUC = $0.989,95 \%$ CI $0.954-1.024 ; p<0.001)$ and in the DWM (AUC $=1,95 \%$ CI $1-1 ; p<0.001)$. The cut-off points were $\geq 13 \% / \mathrm{HPF}$ in the SWM with sensibility $=100.0 \%(95 \% \mathrm{CI}$ $62.88-100.0)$ and specificity $=90.0 \%$ (95\%CI 54.11-99.48) and $\geq 13.6 \% / \mathrm{HPF}$ in the DWM with sensibility $=100.0 \%$ (95\%CI 62.88-100.0) and specificity $=100.0 \%$ (95\%CI $65.55-$ $100.0)$, respectively.

The neocortical markers of acute and chronic neuronal activity could also discriminate the patients with HS (c-fos$\mathrm{AUC}=0.778,95 \% \mathrm{CI} 0.563-0.992, p=0.041 ; \Delta \mathrm{FosB}-\mathrm{AUC}$ 

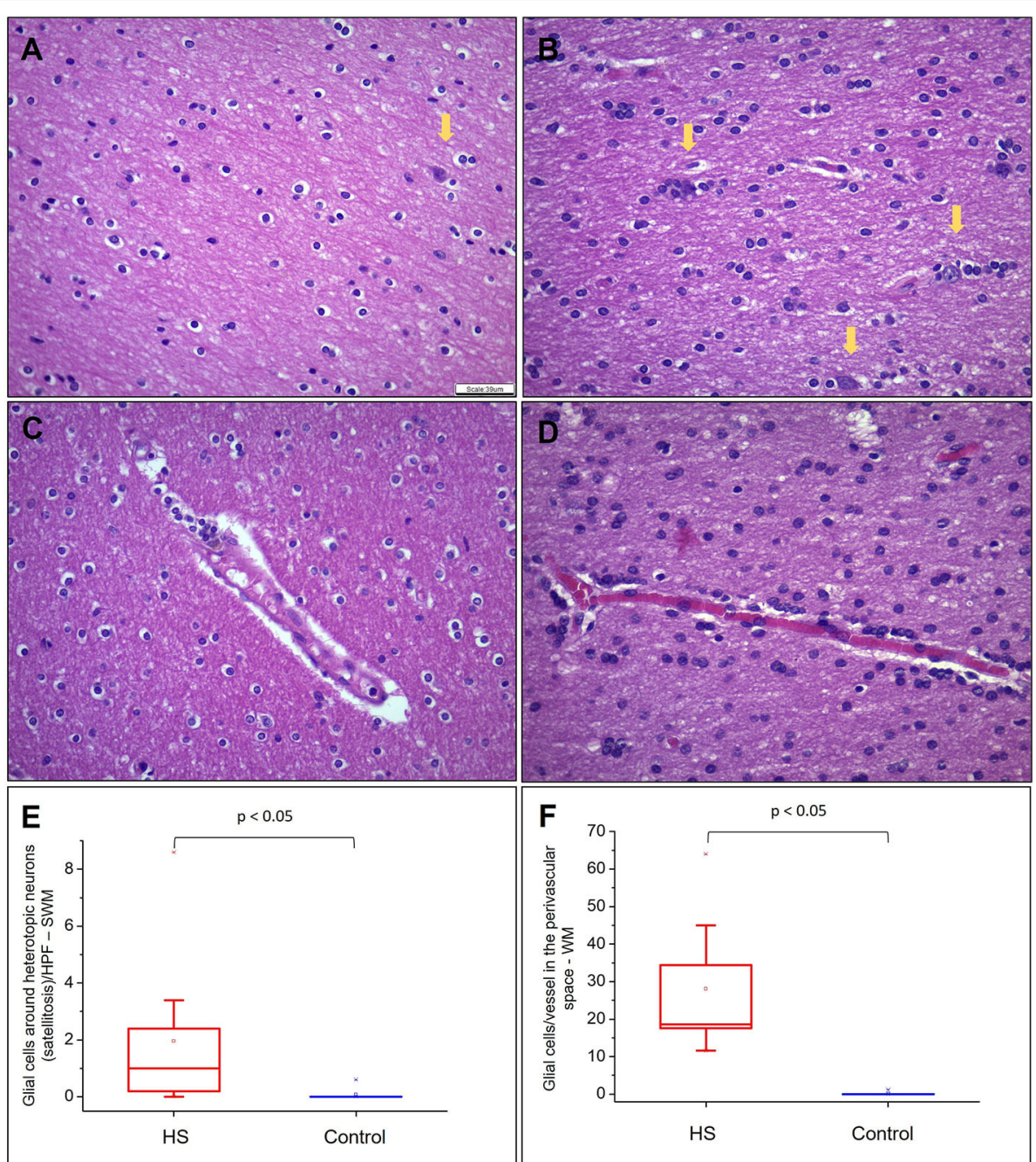

FIGURE 5 | Quantitative analysis of heterotopic neurons, satellitosis, and glial cells in perivascular space in WM. (A) and (B) represent fields photographed in the SWM of a control subject and an HS patient, respectively. Heterotopic neurons are indicated with yellow arrows. (C) and (D) represent glial cells in the perivascular space in a control group and an HS group, respectively. In (E), graphical representation of the mean number of oligodendroglial cells around heterotopic neurons by HPF in SWM. In (F), mean number of glial cells in the perivascular space/vessel in each studied group. Hematoxylin and eosin (A-D). Scale bar: $39 \mu \mathrm{m}$ (A-D).
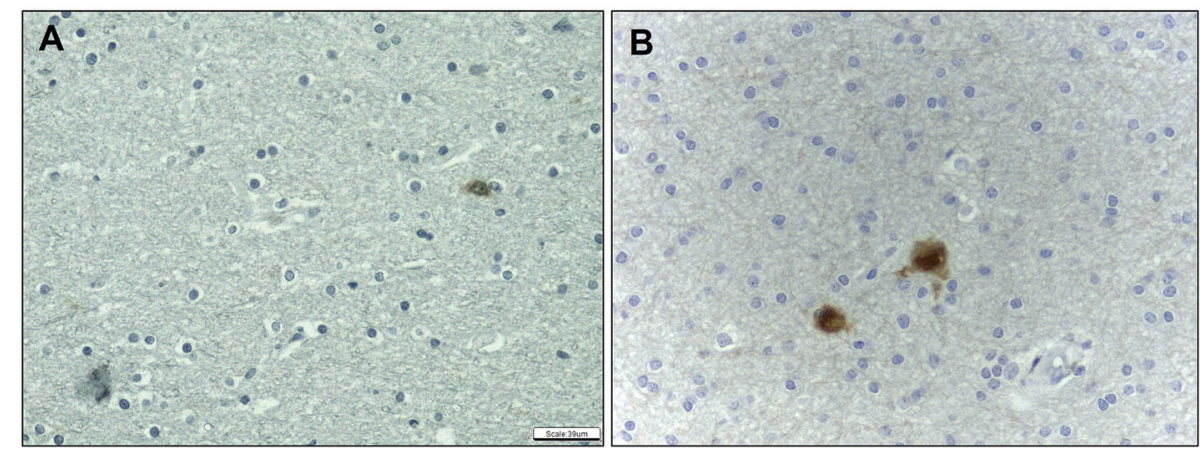

FIGURE 6 | Quantitative analysis of the immunohistochemical expression of NeuN antibody. NeuN expression in control (A) and HS (B) individuals. Peroxidase (A, B) Scale bar: $39 \mu \mathrm{m}$ (A, B). 

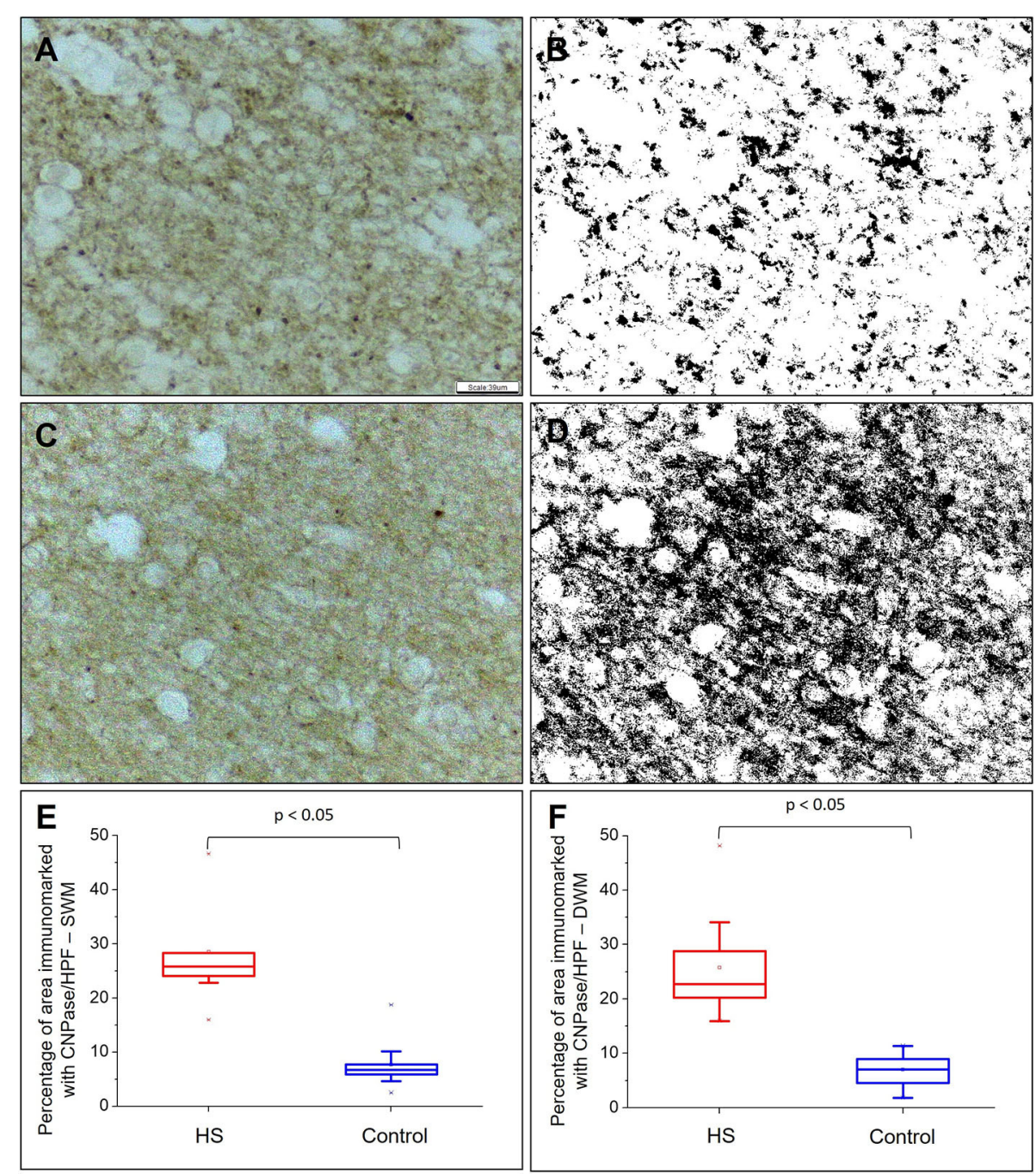

FIGURE 7 | Quantitative analysis of myeloarchitecture through CNPase expression. CNPase expression without counterstaining in control (A) and epilepsy (C) groups. In (B) and (D), transformation of images (A) and (C) are shown, respectively, in gray scale to increase binary contrast (thresholding) and analysis of the percentage of immunomarked area using the ImageJ® program. Graphic representation of the mean percentage of CNPase expression per high power field (HPF) in superficial white matter (E) and deep white matter (F). Peroxidase (A, C). Scale bar: $39 \mu \mathrm{m}$ (A-D)

$=1,95 \%$ CI $1-1, p<0.001)$. For $c$-fos, the cut-off point was $\geq 0.15$ cells/HPF with sensibility $=66.67 \%$ (95\%CI 30.92-90.96) and specificity $=80.0 \%(95 \% \mathrm{CI} 44.22-96.46)$. As for $\triangle \mathrm{FosB}$, the cut-off point was $\geq 3.0$ cells/HPF with sensibility $=100.0 \%$ $(95 \%$ CI $62.88-100.0)$ and specificity $=100.0 \%$ (95\%CI $65.55-$ 100.0).

The T2 signal by relaxometry significantly differentiated individuals with HS from controls (AUC $=0.926$, 95\%CI $0.809-$ $1.043, p=0.002$ ), cut-off being $\geq 70.6$ with sensibility $=88.89 \%$ (95\%CI 50.67-99.42) and specificity $=77.78 \%$ (95\%CI $40.19-$ 96.05).

The FA could also significantly discriminate the HS and control groups (AUC $=0.802,95 \%$ CI $0.555-1.050 ; p=$ $0.031)$. The cut-off was $\leq 0.26$ with sensibility $=100 \%(95 \%$
CI $62.88-100.0)$ and specificity $=77.78 \%(95 \% \mathrm{CI} \quad 40.19-$ 96.05).

\section{DISCUSSION}

This work aimed to investigate and correlate WM alterations in cell populations and neuroimaging from individuals with a common cause of pharmacoresistant epilepsy (HS), and whose surgical treatment allowed the sampling of tissue from the temporopolar region. Specifically, we demonstrated significant changes in WM cellularity when analyzing the anterior temporal pole of patients with HS. Additionally, histopathological alterations of the anterior temporal pole, in particular related 

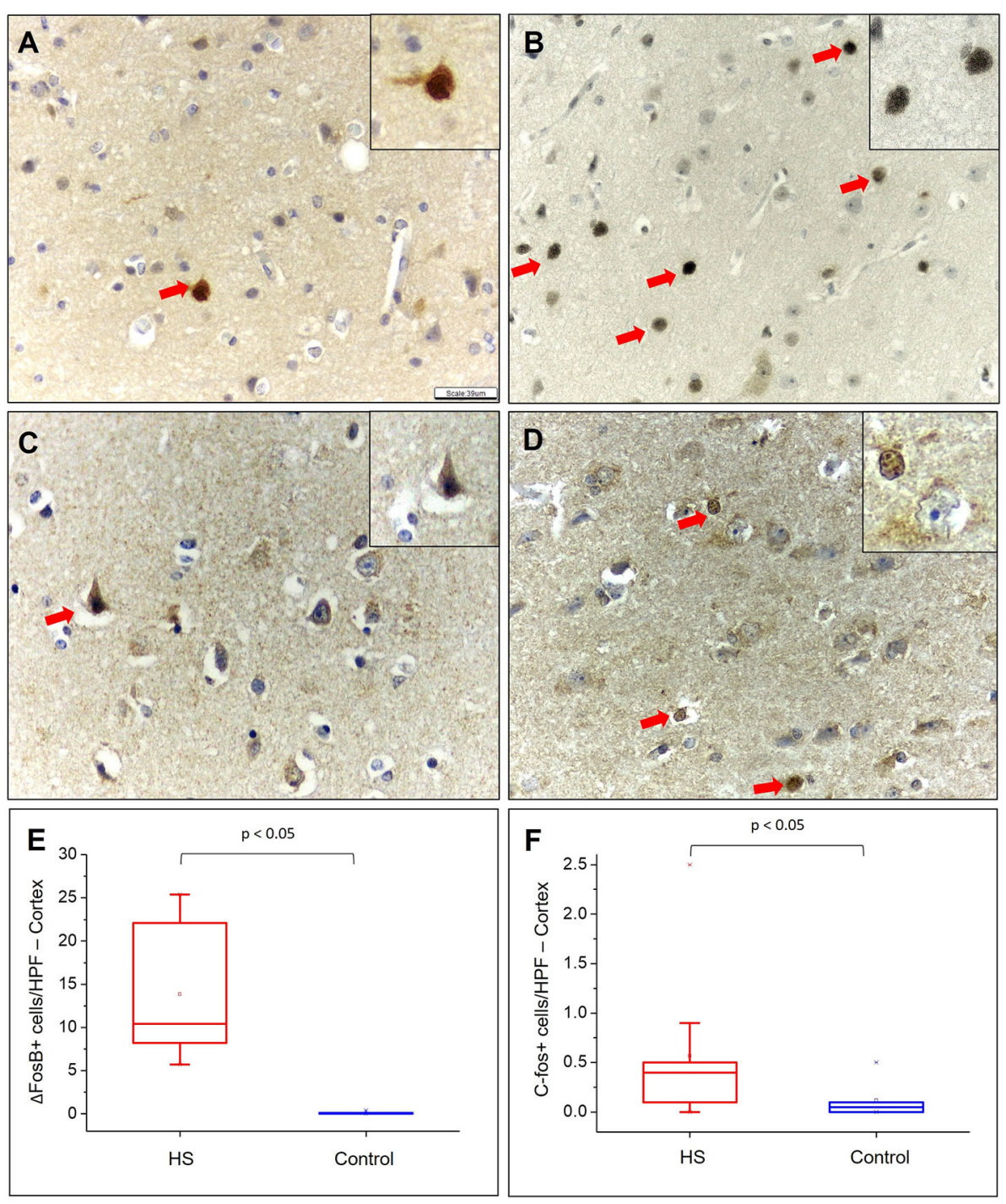

FIGURE 8 | Quantitative analysis of the immunohistochemical expression of $\Delta \mathrm{FosB}$ and c-fos. Expression of $\Delta$ FosB in the cortical region of individuals in the control (A) and HS (B) groups. C-fos expression in the cortical region of individuals in the control group (C) and in the HS group (D). Neurons with expression of $\Delta$ FosB and C-fos are indicated with red arrows. Graphic representation of the average number of $\Delta$ FosB (E) and C-fos (F) positive cells/HPF in neocortex. Peroxidase with hematoxylin counterstaining (A-D). Scale bar: $39 \mu \mathrm{m}$ (A-D).

to the oligodendrocyte population were correlated with MRI findings, allowing to determine cut-off points that could predict changes associated with HS and hypothesize pathophysiological mechanisms for TLE-HS.

Oligodendroglial hypercellularity in the WM was an eyecatching finding in the present investigation. Such a finding has been observed in patients with long-term epilepsy and reported under a wide range of descriptive terms, such as glioneuronal hamartia (30), microdysgenesis $(8,9,31)$, oligodendroglial hyperplasia $(10,15)$, and oligodendrogliosis $(32,33)$. Recently, an entity was proposed in patients with early-onset frontal lobe epilepsy and designated as mild developmental cortical malformation with oligodendroglial hyperplasia $(34,35)$. This myriad of terms has been used for patients with temporal $(8,9$,
$30,36,37)$ or frontal lobe epilepsy $(15,34)$ and associated with different pathological conditions (32).

Changes related to the number and distribution of oligodendrocytes in the WM have been interpreted as a reactive proliferation in the context of pharmacoresistant epilepsy $(32,36)$. Experimental studies on optogenetic stimulation in mice showed evidence supporting this interpretation. Indeed, it was demonstrated that repetitive activation of neurons is capable of stimulating the proliferation of both neuronal and oligodendroglial progenitor cells, their differentiation, and myelination process (11). We verified an increased expression of acute and chronic markers of neuronal activity in the temporal neocortex. This finding suggests that the adaptive neuroplasticity described in experimental models of epilepsy 
TABLE 2 | Summary of the MRI measurements of the anterior temporal lobe in control group and HS group.

\begin{tabular}{|c|c|c|c|}
\hline MRI measurements & Control & HS & $p$-value \\
\hline T2 relaxometry, median (interquartile range) & $68.75(68.71-70.57)$ & $77.51(75.53-86.31)$ & 0.002 \\
\hline FA, median (interquartile range) & $0.39(0.27-0.4)$ & $0.24(0.22-0.243)$ & 0.034 \\
\hline AD, median (interquartile range) & $0.00095(0.00091-0.00122)$ & $0.00121(0.00113-0.00123)$ & 0.185 \\
\hline RD, median (interquartile range) & 0.0007 (0.0005-0.0008) & 0.00088 (0.00079-0.0009) & 0.064 \\
\hline MD, median (interquartile range) & 0.00078 (0.00066-0.00098) & 0.00099 (0.0009-0.00102) & 0.093 \\
\hline
\end{tabular}

can also be observed in human samples from patients with TLE-HS (11, 18, 19). Particularly, as observed in experimental studies (19), the increased expression of $\Delta$ FosB that we verified in patients with TLE could be associated with higher expression of specific subunits of glutamate receptors, thus contributing to seizure generation and propensity to seizures. Moreover, we also verified that increased neuronal activation was positively correlated with oligodendroglial hypercellularity in the WM, even though such increase in cell number was not correlated with disease duration. However, since our patients had more than 21 years of epilepsy duration (ranging from 21 to 50 years), a floor effect should be considered in this particular lack of correlation. Therefore, considering the observations from previous reports on surgical specimens and experimental approaches and the current findings in the temporal pole, it is possible that the alterations related to oligodendroglial cell number and distribution in the WM may be a maladaptive response of the nervous tissue submitted to pharmacoresistant epilepsy, irrespective of etiology.

In this work, the HS group showed an increase in the percentage of the area immunostained for CNPase. This marker is a myelin membrane-associated protein expressed by both oligodendroglial progenitor forms and mature oligodendrocytes, its function being still debatable $(38,39)$. As CNPase is considered to bind to microtubules and to be involved in axonal maintenance, this protein would play a structural role in myelin integrity (40). Therefore, we hypothesize that the increase in the percentage of CNPase immunostained area could be due to fragmentation or structural disorganization of WM fibers in the context of chronic epilepsy $(15,41)$.

Additionally, we demonstrated a significant ipsilateral reduction in FA in the anterior pole of the temporal lobe, corresponding to a decrease in the integrity of the axonal bundles, and increased T2-weighted signal. This result is in line with previous DTI $(42,43)$ and T2-weighted reports $(21,22)$ of widespread changes in TLE-HS affecting not only the hippocampal formation but also other temporal and extratemporal structures. However, fewer studies have investigated the histopathological correlation of WM alterations with imaging in patients with epilepsy.

Overall, FA changes has been more frequently associated with histopathological changes in the SWM. Here, we found that higher numbers of Olig2- and $\Delta$ FosB-positive cells were strongly associated with lower FA values, explaining over $44 \%$ of the variation between these measures. Concha et al. (42) showed lower FA associated with reduced cumulative axonal circumference, axonal density, and myelin thickness and area in the fimbria-fornix tract, thus evidencing the relationship between diffusion microstructure abnormality and underlying axonal pathology. Interestingly, a recent in vivo MRI study (44) also demonstrated that reduced FA was associated with reduced intracellular volume fraction (an MRI marker of neurite density) and myelin water fraction (an MRI marker of myelination). Taken together, it is possible that our findings regarding oligodendrocyte hypercellularity, neocortical neuronal activation, and increase in percentage of CNPase-positive area would suggest that the axonal impairment measured by the FA is part of a complex mechanism involving excessive neuronal activity associated with changes in the myeloarchitecture integrity in patients with TLE.

Moreover, we did not find a significant correlation between T2-weighted signal (relaxometry) and the number of oligodendrocytes in the DWM in hematoxylin and eosinstained sections. In TLE, a hyperintense signal in T2-weighted images has been associated with astrocytic reaction (gliosis) in the hippocampus (45). Therefore, our findings suggest that tissue changes associated with the oligodendrocyte population in the WM may not be the predominant pathophysiological events contributing to changes in T2 hyperintense signal detected in the temporal pole of some patients with TLE. However, these findings need further confirmation.

Our results regarding visual MRI analysis revealed that five out of nine patients presented with temporopolar blurring in T2weighted and FLAIR images. However, the increase in CNPasepositive areas and in the number of oligodendroglial cells (both in hematoxylin and eosin-stained and in Olig2-immunoreacted sections) were similar in patients with and without temporopolar blurring. These findings support that this MRI temporopolar blurring in patients with TLE-HS is most likely related to delayed myelination and arrest of white matter development $(12,46)$ and not predominantly influenced by changes in the number of oligodendrocytes.

The value of hippocampal T2 relaxometry TLE investigation is well-documented using both manual and automatic methods $(47,48)$. Here, we also showed that WM alterations translated by histopathological, FA, and T2 signal changes in the temporal pole allowed for differentiation of patients with HS from controls, with high sensitivity and specificity, pointing out reference cutoffs to indicate pathological changes. In this sense, previous studies have yielded results that allow imaging techniques to anticipate histopathological findings of epileptogenic lesions $(47,49)$. 

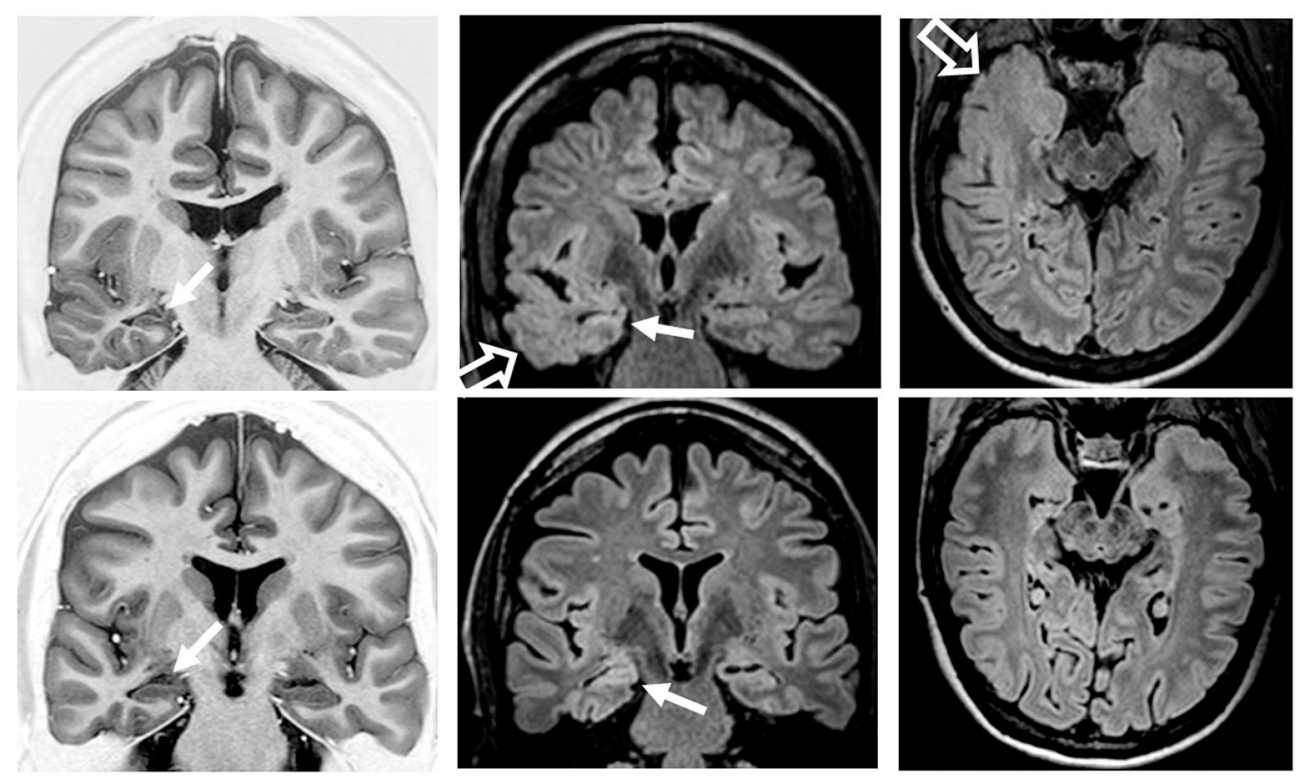

FIGURE 9 | Preoperatory coronal T1-weighted inversion recovery and coronal and axial fluid-attenuated inversion recovery (FLAIR) images from patient 2 (top row) with temporopolar blurring in FLAIR images (open arrows) and from patient 6 (bottom row) without temporopolar blurring. Both patients had MRI signs of right hippocampal sclerosis (atrophy and hyperintense FLAIR signal; arrows).
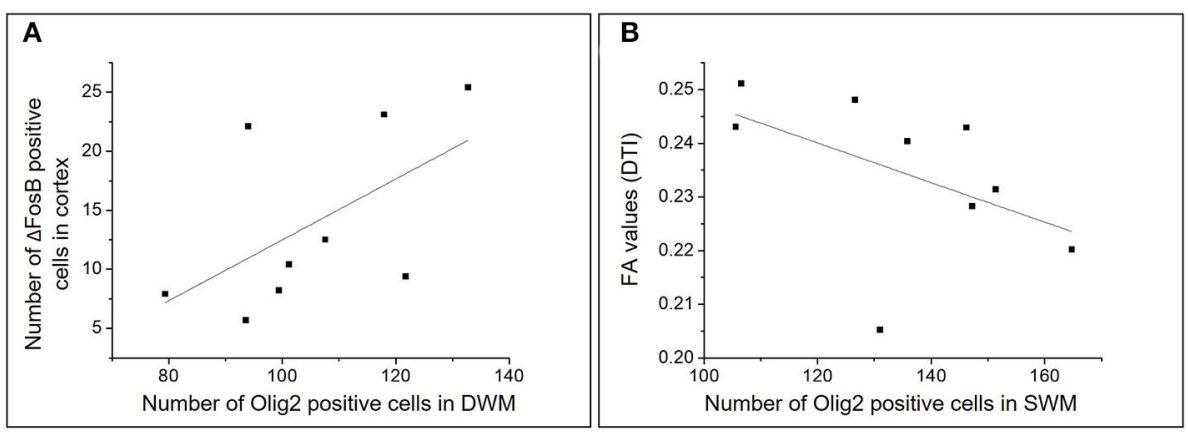

FIGURE 10 | Graphic representations of the analysis of correlations between different numerical variables in the HS group. Scatterplots representing the correlations between the number of $\triangle$ FosB positive cells in the cortex and the number of Olig2 positive cells in the DWM (A) and between the number of Olig2 positive cells in the SWM and the mean FA in individuals with HS (B).

Regarding the limitations of our study, it is important to mention the small sample size of HS and control groups. However, such sample size was homogeneous enough to suggest associations between imaging (T2 and DWI) and neuropathological WM changes. Moreover, further validation of the present histological results could be performed in larger series of surgical cases, including etiologies other than HS. Finally, it could be considered that the influence of fixation in the immunoreactivity of the different types of tissues (surgical and autopsy specimens) in our investigation, an intrinsic limitation of studies in which autopsy samples are used as controls.

In conclusion, after performing histopathological and MRI analyses in the WM of the anterior temporal pole from patients with TLE-HS, we showed changes in cell constitution and myelin remodeling. As we evaluated tissue from individuals with chronic epilepsy, it is possible that the current set of histopathological and quantitative MRI changes is associated with plasticity of neuronal and glial cells of the TLE network. Future studies based on the present pathology-imaging approach may contribute with insights into tissue alterations correlating with MRI signal changes and into pathophysiological mechanisms of other structural lesions causing focal epilepsy.

\section{DATA AVAILABILITY STATEMENT}

The raw data supporting the conclusions of this article will be made available by the authors, without undue reservation. 


\section{ETHICS STATEMENT}

The studies involving human participants were reviewed and approved by Research Ethics Committee of the University of Campinas. The patients/participants provided their written informed consent to participate in this study.

\section{AUTHOR CONTRIBUTIONS}

BZ, IC, BC, LS, CY, FC, and FR: contributed to study design. BZ, IC, BC, LS, VC, KS, BA, MA, HT, CY, EG,

\section{REFERENCES}

1. World Health Organization. Epilepsy Fact Sheet [Internet]. (2019). Available online at: https://www.who.int/news-room/fact-sheets/detail/epilepsy (accessed November 28, 2021).

2. Shorvon SD. The epidemiology and treatment of chronic and refractory epilepsy. Epilepsia. (1996) 37:S1-3. doi: 10.1111/j.1528-1157.1996.tb06027.x

3. Sander JWAS. Some aspects of prognosis in the epilepsies: a review. Epilepsia. (1993) 34:1007-16. doi: 10.1111/j.1528-1157.1993.tb02126.x

4. Kwan P, Arzimanoglou A, Berg AT, Brodie MJ, Hauser WA, Mathern G, et al. Definition of drug resistant epilepsy: consensus proposal by the ad hoc task force of the ILAE commission on therapeutic strategies. Epilepsia. (2010) 51:1069-77. doi: 10.1111/j.1528-1167.2009.02397.x

5. Barba C, Cossu M, Guerrini R, Di Gennaro G, Villani F, De Palma L, et al. Temporal lobe epilepsy surgery in children and adults: a multicenter study. Epilepsia. (2021) 62:128-42. doi: 10.1111/epi.16772

6. Semah F, Picot MC, Adam C, Broglin D, Arzimanoglou A, Bazin B, et al. Is the underlying cause of epilepsy a major prognostic factor for recurrence? Neurology. (1998) 51:1256-62. doi: 10.1212/WNL.51.5.1256

7. Pohlen MS, Jin J, Tobias RS, Maheshwari A. Pharmacoresistance with newer anti-epileptic drugs in mesial temporal lobe epilepsy with hippocampal sclerosis. Epilepsy Res. (2017) 137:56-60. doi: 10.1016/j.eplepsyres.2017.09.012

8. Kasper B, Stefan H, Buchfelder M, Paulus W. Temporal lobe microdysgenesis in epilepsy versus control brains. J Neuropathol Exp Neurol. (1999) 58:228. doi: 10.1097/00005072-199901000-00003

9. Thom M, Sisodiya S, Harkness W, Scaravilli F. Microdysgenesis in temporal lobe epilepsy: a quantitative and immunohistochemical study of white matter neurones. Brain. (2001) 124:2299-309. doi: 10.1093/brain/124.11.2299

10. Burger PC, Scheithauer BW, Vogel FS. Surgical Pathology of the Nervous System and its Coverings. 4th ed. New York, Edinburgh, London, Philadelphia: Churchill Livingstone (2002).

11. Gibson M, Purger D, Mount C, Goldstein A, Lin G, Wood L, et al. Neuronal activity promotes oligodendrogenesis and adaptive myelination in the mammalian brain. Science. (2014) 344:480-1. doi: 10.1126/science.1252304

12. Garbelli R, Milesi G, Medici V, Villani F, Didato G, Deleo F, et al. Blurring in patients with temporal lobe epilepsy: Clinical, high-field imaging and ultrastructural study. Brain. (2012) 135:2337-49. doi: 10.1093/brain/aws149

13. de Souza JPSAS, Pimentel-Silva LR, Ayub G, Nogueira MH, Zanao T, Yasuda $\mathrm{CL}$, et al. Transsylvian amygdalohippocampectomy for mesial temporal lobe epilepsy: comparison of three different approaches. Epilepsia. (2021) 62:43949. doi: $10.1111 /$ epi.16816

14. Blümcke I, Thom M, Aronica E, Armstrong DD, Bartolomei F, Bernasconi $\mathrm{A}$, et al. International consensus classification of hippocampal sclerosis in temporal lobe epilepsy: a task force report from the ILAE commission on diagnostic methods. Epilepsia. (2013) 54:1315-29. doi: 10.1111/epi.12220

15. Schurr J, Coras R, Rössler K, Pieper T, Kudernatsch M, Holthausen H, et al. Mild malformation of cortical development with oligodendroglial hyperplasia in frontal lobe epilepsy: a new clinico-pathological entity. Brain Pathol. (2017) 27:26-35. doi: 10.1111/bpa.12347

16. Herrera DG, Robertson HA. Activation of c-fos in the brain. Prog Neurobiol. (1996) 50:83-107. doi: 10.1016/S0301-0082(96)00021-4
FC, and FR: obtained and analyzed the data. BZ, IC, LS, VC, KS, FC, and FR: drafted the manuscript and figures. All authors contributed to the article and approved the submitted version.

\section{FUNDING}

This study was sponsored by grants from the São Paulo Research Foundation (FAPESP; 2013/075593, 2019/08259-0, 2020/12651-0) and FAEPEX UNICAMP (2037/19).
17. McClung CA, Ulery PG, Perrotti LI, Zachariou V, Berton O, Nestler EJ. DeltafosB: a molecular switch for long-term adaptation in the brain. Mol Brain Res. (2004) 132:146-54. doi: 10.1016/j.molbrainres.2004.05.014

18. Morris TA, Jafari N, Delorenzo RJ. Chronic DeltaFosB expression and increased AP-1 transcription factor binding are associated with the long term plasticity changes in epilepsy. Mol Brain Res. (2000) 79:13849. doi: 10.1016/S0169-328X(00)00112-1

19. Hiroi N, Marek GJ, Brown JR, Ye H, Saudou F, Vaidya VA, et al. Essential role of the fosB gene in molecular, cellular, and behavioral actions of chronic electroconvulsive seizures. J Neurosci. (1998) 18:695262. doi: 10.1523/JNEUROSCI.18-17-06952.1998

20. Mota MVB, Zaidan BC, do Canto AM, Ghizoni E, Tedeschi H, de Souza Queiroz L, et al. ATP synthase subunit beta immunostaining is reduced in the sclerotic hippocampus of epilepsy patients. Cell Mol Neurobiol. (2019) 39:149-60. doi: 10.1007/s10571-018-0641-2

21. Kubota BY, Coan AC, Yasuda CL, Cendes F. T2 hyperintense signal in patients with temporal lobe epilepsy with MRI signs of hippocampal sclerosis and in patients with temporal lobe epilepsy with normal MRI. Epilepsy Behav. (2015) 46:103-8. doi: 10.1016/j.yebeh.2015.04.001

22. Giacomini L, de Souza JPSA, Formentin C, de Campos BM, Todeschini $\mathrm{AB}$, de Oliveira E, et al. Temporal lobe structural evaluation after transsylvian selective amygdalohippocampectomy. Neurosurg Focus. (2020) 48: E14. doi: 10.3171/2020.1.FOCUS19937

23. Lebel C, Walker L, Leemans A, Phillips L, Beaulieu C. Microstructural maturation of the human brain from childhood to adulthood. Neuroimage. (2008) 40:1044-55. doi: 10.1016/j.neuroimage.2007.12.053

24. Campos BM, Coan AC, Beltramini GC, Liu M, Yassuda CL, Ghizoni E, et al. White matter abnormalities associate with type and localization of focal epileptogenic lesions. Epilepsia. (2015) 56:125-32. doi: 10.1111/epi.12871

25. Cendes F. Neuroimaging in investigation of patients with epilepsy. Contin Lifelong Learn Neurol. (2013) 19:62342. doi: 10.1212/01.CON.0000431379.29065.d3

26. Mendes Coelho VC, Morita-Sherman M, Yasuda CL, Alvim MMK, Amorim BJ, Tedeschi $\mathrm{H}$, et al. Magnetic resonance imaging findings and clinical characteristics in mild malformation of cortical development with oligodendroglial hyperplasia and epilepsy in a predominantly adult cohort. Epilepsia. (2021) 62:1429-41. doi: 10.1111/epi.16907

27. Coste S, Ryvlin P, Hermier M, Ostrowsky K, Adeleine P, Froment JC, et al. Temporopolar changes in temporal lobe epilepsy: a quantitative MRI-based study. Neurology. (2002) 59:855-61. doi: 10.1212/WNL.59.6.855

28. Mitchell LA, Jackson GD, Kalnins RM, Saling MM, Fitt GJ, Ashpole $\mathrm{RD}$, et al. Anterior temporal abnormality in temporal lobe epilepsy: a quantitative MRI and histopathologic study. Neurology. (1999) 52:32736. doi: 10.1212/WNL.52.2.327

29. Engel J, Cascino GD, Ness PCV, Rasmussen TB. Outcome With Respect to Epileptic Seizures. In: Surgical Treatment of the Epilepsies. New York: Raven Press (1993). p. 609-21.

30. Wolf HK, Campos MG, Zentner J, Hufnagel A, Schramm J, Elger CEWO. Surgical pathology of temporal lobe epilepsy. Experience with 216 cases. $J$ Neuropathol Exp Neurol. (1993) 52:499-506. doi: 10.1097/00005072-19930900 0-00008 
31. Arai N, Umitsu R, Komori T, Hayashi M, Kurata K, Nagata J, et al. Peculiar form of cerebral microdysgenesis characterized by white matter neurons with perineuronal and perivascular glial satellitosis: a study using a variety of human autopsied brains. Pathol Int. (2003) 53:34552. doi: 10.1046/j.1440-1827.2003.01480.x

32. Sakuma S, Halliday WC, Nomura R, Baba S, Sato Y, Okanari K, et al. Increased subcortical oligodendroglia-like cells in pharmacoresistant focal epilepsy in children correlate with extensive epileptogenic zones. Epilepsia. (2016) 57:2031-8. doi: 10.1111/epi.13590

33. Rodríguez-cruces $\mathrm{R}$, Concha L. White matter in temporal lobe epilepsy : clinico-pathological correlates of water diffusion abnormalities. Quant Imaging Med Surg. (2015) 5:264-78. doi: 10.3978/j.issn.2223-4292.2015. 02.06

34. Hartlieb T, Winkler P, Coras R, Pieper T, Holthausen H, Blümcke I, et al. Agerelated MR characteristics in mild malformation of cortical development with oligodendroglial hyperplasia and epilepsy (MOGHE). Epilepsy Behav. (2019) 91:68-74. doi: 10.1016/j.yebeh.2018.07.009

35. Blümcke I, Coras R, Busch RM, Morita-Sherman M, Lal D, Prayson R, et al. Toward a better definition of focal cortical dysplasia: an iterative histopathological and genetic agreement trial. Epilepsia. (2021) 62:141628. doi: 10.1111/epi.16899

36. Stefanits H, Czech T, Pataraia E, Baumgartner C, Derhaschnig N, Slana A, et al. Prominent oligodendroglial response in surgical specimens of patients with temporal lobe epilepsy. Clin Neuropathol. (2012) 31:40917. doi: $10.5414 / \mathrm{NP} 300536$

37. Emery JA, Roper SN, Rojiani AM. White matter neuronal heterotopia in temporal lobe epilepsy: a morphometric and immunohistochemical study. J Neuropathol Exp Neurol. (1997) 56:1276-82. doi: 10.1097/00005072-199712000-00002

38. Baumann N, Pham-Dinh D. Biology of oligodendrocyte and myelin in the mammalian central nervous system. Physiol Rev. (2001) 81:871927. doi: 10.1152/physrev.2001.81.2.871

39. Kuhn S, Gritti L, Crooks D, Dombrowski Y. Oligodendrocytes in development, myelin generation and beyond. Cells. (2019) 8:1424. doi: $10.3390 /$ cells 8111424

40. Raasakka A, Kursula P. The myelin membrane-associated enzyme 2',3'cyclic nucleotide $3^{\prime}$-phosphodiesterase: on a highway to structure and function. Neurosci Bull. (2014) 30:956-66. doi: 10.1007/s12264-0131437-5

41. Donkels C, Peters M, Fariña Núñez MT, Nakagawa JM, Kirsch M, Vlachos A, et al. Oligodendrocyte lineage and myelination are compromised in the gray matter of focal cortical dysplasia type IIa. Epilepsia. (2020) 61:17184. doi: 10.1111/epi.16415

42. Concha L, Livy DJ, Beaulieu C, Wheatley BM, Gross DW. In vivo diffusion tensor imaging and histopathology of the fimbria-fornix in temporal lobe epilepsy. J Neurosci. (2010) 30:996-1002. doi: 10.1523/JNEUROSCI.1619-09.2010
43. Hatton SN, Huynh KH, Bonilha L, Abela E, Alhusaini S, Altmann $A$, et al. White matter abnormalities across different epilepsy syndromes in adults: an ENIGMA-Epilepsy study. Brain. (2020) 143:2454-73. doi: 10.1093/brain/awaa200

44. Winston GP, Vos SB, Caldairou B, Hong SJ, Czech M, Wood TC, et al. Microstructural imaging in temporal lobe epilepsy: diffusion imaging changes relate to reduced neurite density. NeuroImage Clin. (2020) 26:102231. doi: 10.1016/j.nicl.2020.102231

45. Briellmann RS, Kalnins RM, Berkovic SF, Jackson GD. Hippocampal pathology in refractory temporal lobe epilepsy: T2-weighted signal change reflects dentate gliosis. Neurology. (2002) 58:26571. doi: 10.1212/WNL.58.2.265

46. Prado CAC, Federico P, Bernasconi A, Bernhardt B, Caciagli L, Concha L, et al. Imaging characteristics of temporopolar blurring in the context of hippocampal sclerosis. Epileptic Disord. (2021). doi: 10.1684/epd.2021.1378. [Epub ahead of print].

47. Coan AC, Kubota B, Bergo FPG, Campos BM, Cendes F. 3T MRI quantification of hippocampal volume and signal in mesial temporal lobe epilepsy improves detection of hippocampal sclerosis. Am J Neuroradiol. (2014) 35:77-83. doi: 10.3174/ajnr.A3640

48. Winston GP, Vos SB, Burdett JL, Cardoso MJ, Ourselin S, Duncan JS. Automated T2 relaxometry of the hippocampus for temporal lobe epilepsy. Epilepsia. (2017) 58:1645-52. doi: 10.1111/epi.13843

49. Bartolini E, Cosottini M, Costagli M, Barba C, Tassi L, Spreafico R. Ultra-high-field targeted imaging of focal cortical dysplasia : the intracortical black line sign in type IIb. Am J Neuroradiol. (2019) 40:213742. doi: 10.3174/ajnr.A6298

Conflict of Interest: The authors declare that the research was conducted in the absence of any commercial or financial relationships that could be construed as a potential conflict of interest.

Publisher's Note: All claims expressed in this article are solely those of the authors and do not necessarily represent those of their affiliated organizations, or those of the publisher, the editors and the reviewers. Any product that may be evaluated in this article, or claim that may be made by its manufacturer, is not guaranteed or endorsed by the publisher.

Copyright (c) 2021 Zaidan, Cardoso, Campos, Silva, Coelho, Silveira, Amorim, Alvim, Tedeschi, Yasuda, Ghizoni, Cendes and Rogerio. This is an open-access article distributed under the terms of the Creative Commons Attribution License (CC BY). The use, distribution or reproduction in other forums is permitted, provided the original author(s) and the copyright owner(s) are credited and that the original publication in this journal is cited, in accordance with accepted academic practice. No use, distribution or reproduction is permitted which does not comply with these terms. 